\title{
A catalog of M-type star candidates in the LAMOST data release 1
}

\author{
Jing Zhong ${ }^{1}$, Sébastien Lépine ${ }^{2}$, Jing Li $^{1}$, Li Chen $^{1}$, Jinliang Hou ${ }^{1}$ \\ ${ }^{1}$ Key Laboratory for Research in Galaxies and Cosmology, Shanghai Astronomical \\ Observatory, Chinese Academy of Sciences, 80 Nandan Road, Shanghai, China \\ email:jzhong@shao.ac.cn \\ ${ }^{2}$ Department of Physics \& Astronomy, Georgia State University, 25 Park Place, Atlanta, \\ GA 30303, USA
}

\begin{abstract}
In this work, we present a set of M-type star candidates selected from the LAMOST DR1. A discrimination method with the spectral index diagram is used to separate $M$ giants and $\mathrm{M}$ dwarfs. Then, we have successfully assembled a set of $\mathrm{M}$ giants templates from M0 to M6, using the spectra identified from the LAMOST spectral database. After combining the M dwarf templates in Zhong et al. (2015a) and the new created M giant templates, we use the M-type spectral library to perform the template-fit method to classify and identify M-type stars in the LAMOST DR1. A catalog of M-type star candidates including $8639 \mathrm{M}$ giants and 101690 $\mathrm{M}$ dwarfs/subdwarfs is provided. As an additional results, we also present other fundamental parameters like proper motion, photometry, radial velocity and spectroscopic distance.
\end{abstract}

Keywords. stars: fundamental parameters — stars: late-type — catalogs — surveys

\section{Overview of the M-type spectral templates}

In our previous work (Zhong et al. 2015a), a set of M dwarf templates has been developed as references for automatically identifying and classifying the $M$ dwarfs in the LAMOST spectroscopic data. With these well defined $M$ dwarf templates, the templatefit method was used to determine the spectral type of LAMOST stars. As we described in Zhong et al. (2015a), although our M dwarf templates provide a reliable estimate of spectral classification, a fraction of $\mathrm{M}$ giants are mis-classified because we lack $\mathrm{M}$ giant templates in our spectral library. To solve this problem, we created a new M-type spectral templates library by combining the $\mathrm{M}$ dwarf/subdwarf templates and the $\mathrm{M}$ giants templates.

Since the surface gravity is totally different for giants and dwarfs, one can use the spectral features as gravitational indicators to determine the luminosity classes. First, we used the template-fit method to select M-type spectra which positively present the characteristic molecular features. Then the spectral indices of $\mathrm{TiO} 5, \mathrm{CaH} 2$ and $\mathrm{CaH} 3$, as defined by Reid et al. (1995) and Lépine et al. (2007), were calculated. Figure 1 shows the spectral indices diagram for all $M$ type stars we identified in the LAMOST DR1. Two populations are clearly distinguishable in this spectral indices diagram. Giants with weaker $\mathrm{CaH}$ molecular bands are located on the upper branch, which is consistent with the giant/dwarf discrimination by Mann et al. (2012).

As shown in Covey et al. (2007), the SDSS $r-i$ color for late-type stars has shown good relationship with the Morgan-Keenan (MK) spectral subtypes, which spans about 2 mag from M0 to M10. To provide spectral subtypes along the temperature sequence for M giants, we choose the SDSS $r-i$ color as an indicator to classify M giant subtypes. Then, a set of criteria were adopted to select high quality LAMOST spectra as training spectra for each spectral subtype grid. Finally, approximately 200 high quality giant 


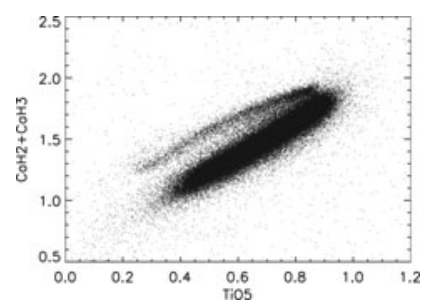

Figure 1. The $\mathrm{M}$ type stars distribution in the $\mathrm{CaH} 2+\mathrm{CaH} 3$ against TiO5 diagram. Two branches in this diagram clearly indicate the two populations. Because of the weaker $\mathrm{CaH}$ molecular bands, about $10000 \mathrm{M}$ giants are located in the upper branch. Comparison with previous results, Lépine et al. (2007) shows that the stars distributed in the lower branch are mainly $\mathrm{M}$ dwarfs/subdwarfs. The clear separation of different populations in this diagram indicate the great potential of using spectral indices to distinguish $\mathrm{M}$ giants and $\mathrm{M}$ dwarfs.

spectra with good photometry in SDSS were left as the training spectra to assemble a grid of temperature sequence. Since in our sample there is no giant candidate with $r-i$ color greater than $2.0 \mathrm{mag}$, the synthetic M giant templates span the spectral subtypes from M0 to M6.

To correct the radial velocity for each training spectrum, we manually used the IRAF package to measure the wavelength correction to the zero-velocity rest-frame. For each spectral subtype bin, at least five training spectra were combined to create the synthetic template spectra.

In the whole M-type templates, there are $\mathrm{M}$ dwarf templates with temperature from K7.0 to M8.5 and metallicity from dMr to usdMp, and the M giant templates from M0 to M6. The total number of M type templates is 223 .

\section{Results}

Based on the M-type templates, we re-run our spectral classification pipeline (Zhong et al. 2015a) to automatically identify and classify M-type stars with spectra from the LAMOST DR1 data. In the classification pipeline, the template-fit method is used by calculating the chi-square values between the LAMOST spectrum and each of the template spectra. Then, the template spectrum which has the minimum chi-square value is considered as the best-fit, and its spectral subtype is used to mark the corresponding LAMOST spectrum.

After passing through the 2,204,696 LAMOST DR1 spectra to our spectral classification pipeline, we identified $8639 \mathrm{M}$ giants and 101,690 M dwarfs/subdwarfs. The excluded spectra were marked as non-M type spectra of which most are earlier type objects like AFGK stars, and a small fraction of spectra were too noisy to be classified.

Finally, we have successfully assembled a set of $\mathrm{M}$ giant templates from M0 to M6 by using the LAMOST DR1 spectra. The M-type star candidates are cataloged in Zhong et al. (2015b). We present the information of celestial coordinates, $\mathrm{JHK}_{s}$ infrared magnitudes in 2MASS, spectral subtypes, radial velocity and derived spectroscopic distance.

\section{References}

Covey, K. R., Ivezić, Ž., Schlegel, D., et al. 2007, AJ, 134, 2398

Lépine, S., Rich, R. M., \& Shara, M. M. 2007, ApJ, 669, 1235

Mann, A. W., Gaidos, E., Lépine, S., \& Hilton, E. J. 2012, ApJ, 753, 90

Reid, I. N., Hawley, S. L., \& Gizis, J. E. 1995, AJ, 110, 1838

Zhong, J., Lépine, S., Hou, J., et al. 2015, AJ, 150, 42

Zhong, J., Lépine, S., Li, J., et al. 2015, RAA, 15, 1154 